\title{
FEL Methodology and Dendograms: Graphic Analysis of Project Activities with Deviations of Operations in Steel Plants
}

\author{
Iandra Vasconcelos de Castro Rodrigues, Mariana Dias Gomes, Nicole Mendanha do Couto, \\ Renata Duarte Mellim, Luiz Melk de Carvalho, Diva de Souza e Silva Rodrigues, Flávio \\ Henrique Batista de Souza
}

${ }^{1}$ Centro Universitário de Belo Horizonte - UNIBH, Brazil

\begin{abstract}
.
Through project management, the use of the FEL (Front-End Loading) methodology was analyzed for project planning and control, in a steel plant located in Belo Horizonte (city of more than 3 million inhabitants). The values presented refer to a real study of strategic adequacy. In order to reduce the costs and deadlines, a method was developed that ensures greater chances of success and accuracy for planning the activities of the teams by grouping tasks with dendograms. The evaluation via dendograms includes groups of activities separated by activity disciplines. With the proposed technique, it is possible to view delay times, activities that need attention, as well as departments that need monitoring. The proposal became viable and effective in projects whose execution parameters are months (or years), which reach thousands (or even millions) of Brazilian Reais for a company, contributing to the survival and/or growth on the market. As a contribution this research demonstrates: an evaluation of the delivery time deviations from the activities of a real project, estimated for 180 days, with 627 activities, between the years of 2018 and 2019, with delivery time deviations between 30 to $100 \%$ that is equivalent to thousands of Brazilian Reais; a process of adapting activities, using the FEL methodology to reorganize the procedures; a methodology to analyze deviations and groups of critical activities, through dendograms, with various linkage techniques, to monitor delays and deviations in deliveries.
\end{abstract}

Keywords: Dendograms, Steel Plants, Management, Projects, Front-End Loading

\section{Introduction}

Project management directly impacts the final quality of a product. With the implantation of a methodology based on the grouping of tasks via a dendogram, it is possible not only to monitor similar activities for the duration, but sectors responsible for executions, in projects that (even with the prerogative project management methodologies) has deviations.

According to Neto et al. (2018), the PMI (Project Management Institute) defines a project as a set of temporary activities, carried out in groups, aimed at producing a single product, service 
or result. Applying the FEL (Front End Loading) methodology, also known as phase-gates, which is a project management technique in which a project is divided into phases, that at the end of each stage there is a decision gate (gate) where the decision makers may consider whether or not to continue with the project. However, it is a method that requires more study time before its initiation, increasing the return on investment.

According to Murtagh \& Contreras (2017), cluster analysis, or clustering, is the name given to the group of computational techniques whose purpose is to separate objects into groups, based on the characteristics that these objects have. The basic idea is to place objects in the same group that are similar according to some predetermined criteria. The criterion is usually based on a dissimilarity function, which takes two objects and returns the distance between them.

Therefore, the objective of this work is to carry out an analysis of the use of the FEL methodology, in a large steel plant in the city of Belo Horizonte (a city of more than $3,000,000$ inhabitants) and to propose the use of the developed grouping method for managing project activities, which allows the extraction of characteristics from these data, separating them into functional groups or hierarchizing them, in order to identify (and possibly reduce) production deviations.

As specific objectives of this research, the following can be mentioned: to carry out an evaluation on the current project management method; evidence obtained results; and to propose a new methodology for analysis of dissimilarity via dendogram analysis with the use of the FEL methodology, to group tasks and monitor deviations.

The justification for this work is supported by the need for viable project management solutions, which are desired by organizations that want to remain in the market. In addition to the fact that with the technique employed, an automated analysis of similarity of activities, can conduct investments and risk analysis with numerical basis.

A similar methodology, using dendograms in a Brazilian company, has already been carried out by Pinto et al (2014). Their proposal presents a collection of variables and the activities of a current project. Thus, an analysis is made on the dissimilarity between them. Recently, other works were carried out with the same intention, in Brazilian companies. In these cases, the use of graphic tools to analyze similarity on project activities, such as Dantas (2018) and Moraes \& Laurindo (2018). However, an analysis is not carried out with groupings of activities according to a hierarchical level of activity, nor is an analysis presented in relation to a project that followed a project management methodology (FEL for example). In addition to the fact that this research focuses on a project with deviations from the deadline (and consequently other resources).

\section{Methods}

\subsection{Dendogram}

The dendrogram, also known as a tree graph or phenogram, is the graphical representation of a grouping hierarchy, and can present one or more cluster solutions. To visualize a grouping process, step by step, and analyze the levels of distances between these clusters, the dendrogram is used, which in turn can be represented in two ways: vertically or horizontally, 
in order to graphically synthesize the analyzes of the information for treatment and discussion of groupings (Murtagh \& Contreras, 2017).

\subsubsection{Clustering Analysis.}

Cluster analysis is a useful tool for checking data in many different situations. This technique can be used to reduce the size of a data set, reducing a wide range of objects to the information at the center of the data set. Bearing in mind that linkage or clustering is an unsupervised learning technique (it occurs when the process is called classification), it can also be used to extract hidden characteristics from the data and develop hypotheses about its nature.

\subsubsection{Hierarchical Algorithms}

According to Murtagh \& Contreras (2017), hierarchical algorithms create a hierarchy of relationships between elements. The agglomerative version, which operates by creating sets from functional elements, works according to the following steps:

1. Make a grouping for each element;

2. Find the most similar pairs of clusters, according to a chosen distance measure;

3. Merge them into a larger grouping and recalculate the distance from this cluster to all other elements;

4. Repeat steps 2 and 3 until a single cluster remains.

According to Shmueli et al., 2017, there are three different ways to measure the distance between two groups, which are described in the following topics and exemplified in figure 1.

- Single Linkage: it is an agglomerative method of hierarchical grouping in which new groups are created by joining the most similar groups. The initial grouping is formed only by singletons, and at each iteration of the method a new group is formed by joining the two most similar groups from the previous iteration. In this method, the distance (proximity) between the new group and the others is determined as the shortest distance between the elements of the new group and the remaining groups.

Mathematically, the link function, distance $D$ between groups $g_{1}$ and $g_{2}$, is described by expression (1):

$D\left(g_{1}, g_{2}\right)=\min (d(x, y))$

where $d(x, y)$ is the distance between the elements $x$ and $y$, and $\theta_{1}$ and $\theta_{2}$ are two groups.

- Average ou Centroid Linkage: the distance is given by the distance between its centroid. The problem is to recalculate centroid and distance between clusters each time a group changes.

- Complete Linkage: it operates similarly to the single linkage, but the distance of the new group to the others is calculated as the maximum distance between the elements of the new group remaining. Mathematically, the link function, distance $D\left(g_{1}+g_{2}\right)$ between groups $g_{1}$ and $g_{2}$, is described by expression (2): 


$$
D\left(g_{1}, g_{2}\right)=\max (d(x, y))
$$

where $d(x, y)$ is the distance between the elements $x$ and $y$, and $g_{1}$ and $g_{2}$ are two groups.

The distance between groups is the distance between their most distant points. There is a divisive version of the hierarchical grouping algorithm, where this version begins with a single grouping containing all the elements and begins to divide it (Murtagh \& Contreras, 2017).

In each iteration, a flat algorithm is used, in order to separate the current set into smaller clusters, repeating the process recursively until there are only sets composed of a single element or until a previously established stop criterion is met . Note that it is usual to divide the selected grouping into two smaller groups, as this makes this technique appear to be the inverse of agglomerative algorithms. However, the number of generated clusters does not necessarily have to be two. One way to choose the number of clusters to be generated is to use a cut cost optimization metric.

It uses two fundamental costs, called intra-cluster similarities and extra-cluster similarities and seeks to maximize the relationship between the two in order to obtain the partitioning in which the generated clusters have greater cohesion.

Figure 1. Distance measurements between two clusters for use in hierarchical algorithms. (a) single linkage, where the distance between groups is given by the distance between their closest points. (b) average linkage, where the distance between groups is given by the distance between their centroid and (c) complete linkage, where the distance between groups is given by the distance between their most distant points

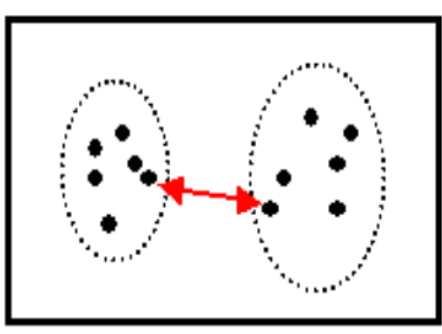

(a)

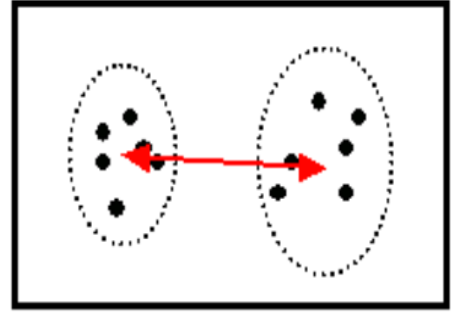

(b)

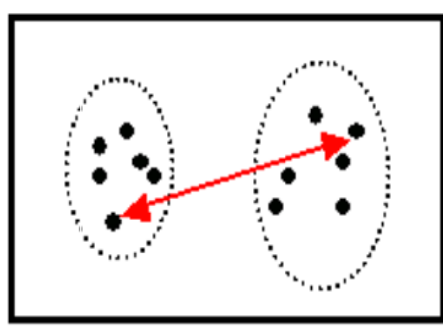

(c)

Source: (Shmueli et al., 2017 - Adapted)

Mathematically it has (Shmueli et al., 2017):

- $V=\left\{v_{1}, v_{2}, \ldots, v_{n}\right\}$ as the set of all existing objects;

- $\left\{C_{1}, C_{2}, \ldots, C_{k}\right\} \mid V=\mathrm{U}_{i=1}^{k} C_{i}$, partitioning of objects, then:

Intra-cluster similarity given by:

$\operatorname{intra}_{p}=\sum_{v_{i}, v_{j} \in C_{p}} \operatorname{sim}\left(v_{i}, v_{f}\right)$ 
Extra-cluster similarity given by:

$$
\operatorname{extra}_{p}=\sum_{v_{i} \in C_{\bar{P}} v_{j} \notin C_{\bar{P}}} \operatorname{sim}\left(v_{i}, v_{j}\right)
$$

Then, the cost of partitioning with $k$ sets is given by:

$$
\text { cust } o_{k}=\sum_{p=1,2, m i k} \frac{\text { intra }}{\text { extra } a_{p}}
$$

Finally choosing the partitioning for which the cost value is as low as possible.

\subsection{Experimental Methodology}

The present paper is characterized as a case study and an experimental analysis, since it interacts with the evaluation of a real case added to the experiment of an analysis proposal. During the research, an analysis was first made about a real and completed project.

The project itself was defined, its dates of occurrences, an analysis of its deviations and nonconformities was carried out. Then an analysis of the project management methodology used for an assessment of which method and how it was employed on the project.

Finally, a methodology is demonstrated, which is the proposal of this paper, based on a dendrogram of representation and automated graphic analysis on the similarity of activities, focusing on a project with deviations and monitoring of responsible times and sectors.

\section{Results}

\subsection{Project Analysis}

\subsubsection{Scope and Project}

The chosen project is based on a demand that emerged in 2014, where the company was audited and prohibited the operation of some machines due to non-compliance with the safety standard for equipment and machines - NR12, requiring their regularization. These machines were at odds, because they allow people to access moving parts during the operating cycle without the controls required by legal requirements. The legislation requires fixed and mobile protections monitored by the safety interface to access the equipment's risk zone in a safe manner. So the project was defined with the following objectives:

- Purchase and installation of a security system to monitor and block equipment.

- Purchase and installation of a light tower to indicate the status of the area (safe or in operation).

- Software development and interface with a supervisory system for diagnosing safety interlocks;

- System commissioning, assisted operation and operation and maintenance training. 
With these premises in mind, the project management methodology to be used was defined.

\subsubsection{Analysis of the Methodology Employed}

The methodology used in the project was FEL, under the use of company $\mathrm{X}$ is structured in phases with a logical sequence to ensure a high level of assertiveness during the planning, analysis, approval, execution and budgetary control of the Projects. At the end of each phase (FEL1, FEL2 and FEL3, Execution and Operation), the Project team holds a meeting with the Stakeholders to assess whether the minimum quality requirements and costs of the expected deliverables are in compliance, and also decide whether the Project will be able to proceed to the next phase, if not, it should be revised or discontinued.

Characterized by making use of engineering documentation such as graphics, tables, plans and drawings of process flows, where it seeks to ensure that the final product is within the expected technical specifications, with the plans and costs aligned with the project strategy. To achieve these results, company $X$ used the following documents for FEL 1: Opening statement; Acquisition plan; Profitability calculation.

\subsubsection{Deviation Analysis}

During the critical evaluation process, some questions that serve as a basis for the analysis were delimited. The graph in Figure 2 shows the budget deviations that occurred during the project representing expenses, between planned and realized values, using the FEL methodology, at Company X.

Figure 2: Deviation curve 2018

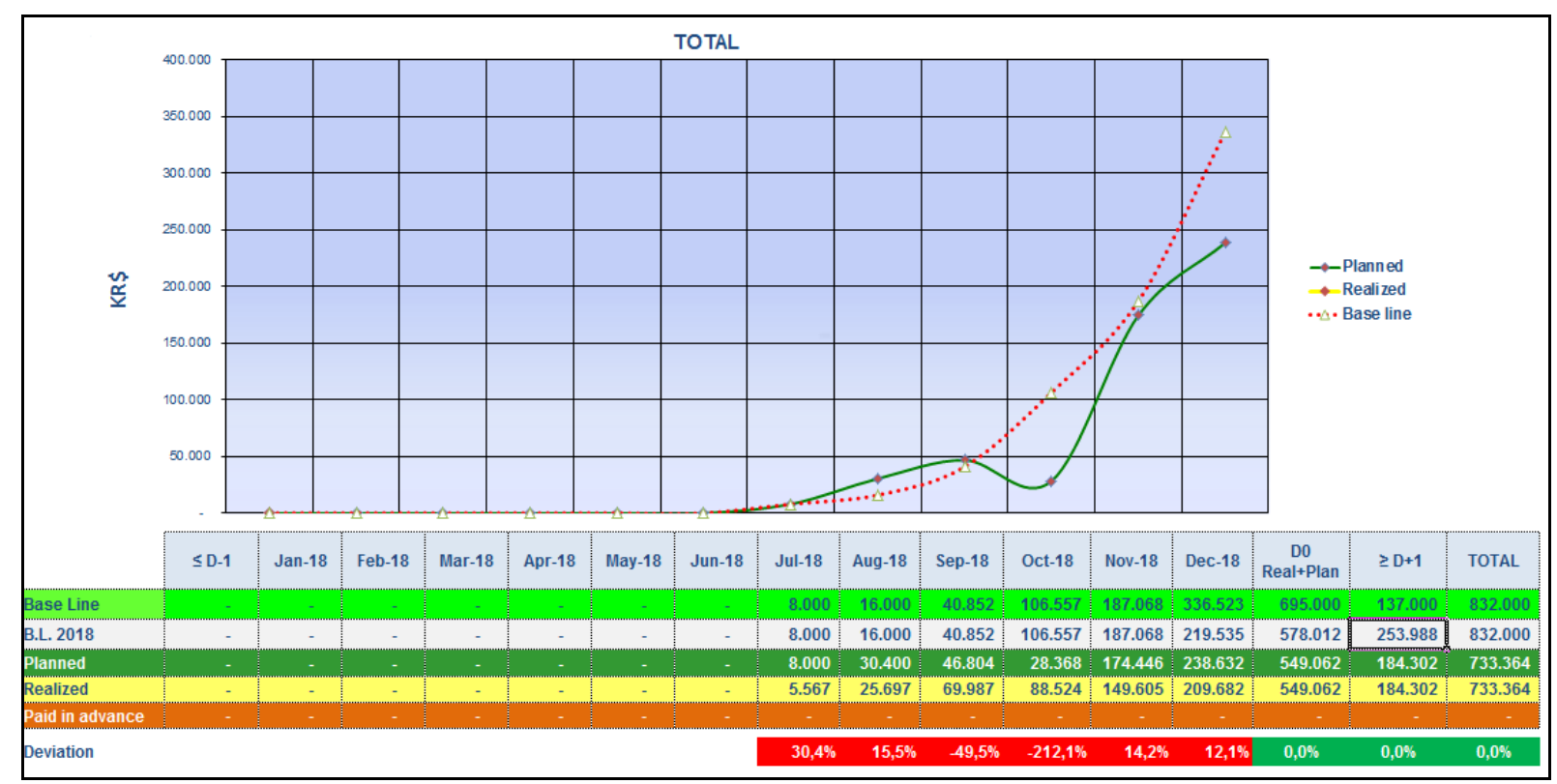

Source: (Authors - Data for the year 2018 provided by Company X) 
Figure 3: Deviation curve 2019

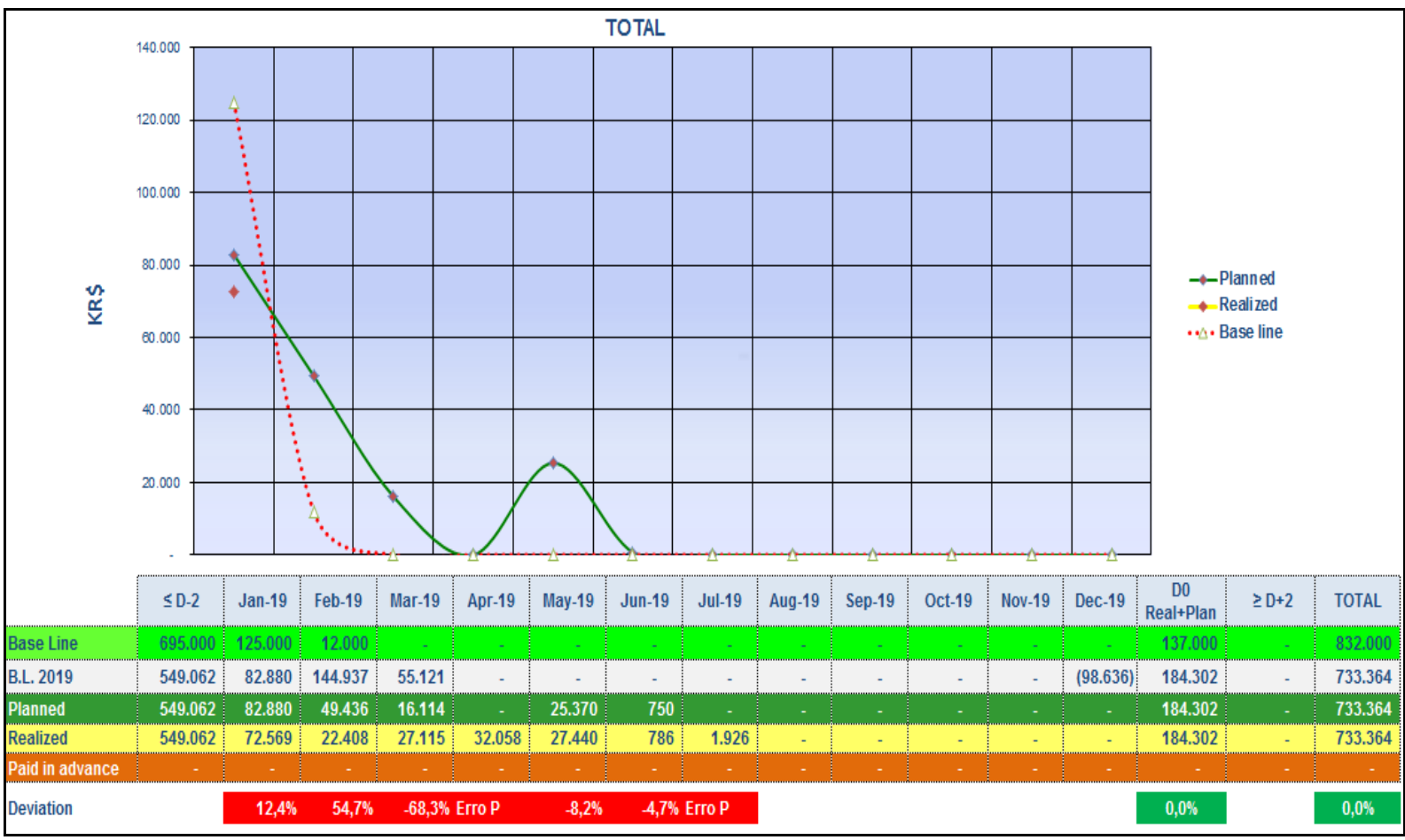

Source: (Authors - Data for the year 2018 provided by Company X)

It is observed that the planned values did not obtain coherence with the realized values, with approximately $30 \%$ deviations in 2018. In 2019, according to the graph in Figure 3, a further $100 \%$ deviation is observed, since it was predicted that the project ended in February 2019, which did not happen, coming to an end only in July 2019.

\subsection{Adaptation Process}

When analyzing the project approval documentation using the FEL methodology, developed by the project team, it was found that it was not used correctly, failing to carry out the FEL 2 (conceptual engineering) and FEL 3 (plan and execute) phases, starting in the FEL 1 phase (identifying and evaluating the need for investment), then execution and closure.

It is observed that when starting the project in the FEL 1 stage, the chances of guaranteeing success in terms of deadlines and costs are reduced and the detailed definition of the undertaking is not so assertive, as there were no plans, studies and analyzes of the best way to project execution.

Even during the execution of the project it could be observed that the deadlines would not be met, as it is a critical project, with a mandatory and emergency feature to be implemented.

The project manager created the schedule and task planning in the Microsoft Project ${ }^{\circledR}$ Software, where the person responsible adjusts the groupings of activities manually, based on his experience, which includes the following information: activity; duration; predecessor and successor tasks. A range of approximately 627 activities were evaluated. 


\subsection{Dendogram Based Methodology Proposal}

In order to reduce costs and deadlines, a method has been developed that guarantees greater chances of success with the use of the FEL methodology and accuracy for planning activities, via grouping of tasks with dendrograms, which calculates mathematically which similar functions will be performed by the same team. Thus the project manager does not need much experience to plan and decide which tasks are directed to which professionals. Firstly, the following assumptions (characteristics) of the data to be used on each activity were defined:

- Activity: Name of the activity, which will not be used for calculations, but for evaluation references;

- Predominant area: determining which engineering sector is predominant in the responsibility for carrying out the activity. Integers were used to code each of the areas (5 were used for analysis), where: $\{1=$ Engineering; $2=$ Electric; 3 = Project Manager; 4 = Mechanics; 5 = Assembly $\}$;

- Duration: duration of the activity in days;

- Disciplinarity: ' 1 ' for just one discipline or ' 2 ' for multidisciplinary, when the activity is to be performed with more than one predominant area;

- Number of Disciplines: integer to say how many areas (in case of multidisciplinarity) are involved (minimum value equal to 1 );

To develop the method, the schedule of activities listed by the project manager of Company $\mathrm{X}$ was used for testing.

\subsubsection{Analysis Without Clusters By Levels Analysis of Deviations}

In the first experiment, the last level of indentation of the activity or task was used for greater information accuracy. Table 1 shows an example of the activities used for experiments, where, in this case, 46 initial project activities were used.

Table 1: Examples of activities without clusters

\begin{tabular}{|l|l|l|l|l|l|}
\hline Activities & Code & Predominant area & duration & Disciplinarity & number of Disciplinarity \\
\hline Mechanical & 1 & 1 & 17 & 2 & 2 \\
\hline Electrical Detailed Engineering & 2 & 2 & 15 & 2 & 2 \\
\hline TA Detailed Engineering & 3 & 1 & 17 & 1 & 1 \\
\hline Readiness Review - Kick-Off & 4 & 1 & 5 & 2 & 5 \\
\hline Readiness Review - Erection & 5 & 1 & 1 & 2 & 2 \\
\hline Electrical Components & 39 & 2 & 42 & 1 & 1 \\
\hline
\end{tabular}

Source: (Authors)

Evaluations were performed using dendrograms to check for dissimilarity. In the first experiment, the Single (Appendix Ia), Average / Centroid (Appendix Ib) and Complete (Appendix Ic) methods were used. Even with different forms of analysis among the options 
for analyzing the dissimilarity between the activities, they were consistent in relation to the proposed grouping.

It can be noted that a group composed of the activities $\{1,2,3,17,18\}$ has a characteristic dissimilarity that separates them from the other 41 activities. In more detail, it can be seen that the activities $\{1,2,3\}$ constitute a subset among the five mentioned, with a greater proximity between them.

In the focus of interpretation of results, with the characteristics taken as a premise (duration and multidisciplinary issues), through numerical and automated means, the manager could, with this methodology, infer that the activities referenced in this first assessment are similar in consumption these resources. Thus, during the determination of these activities in similar projects, he could assess the question of deadlines and the disciplines of the teams to be assigned, with greater assertiveness and with mathematical support, without bias, based on mathematical similarity.

Two caveats should be placed on the agenda regarding the first experiments presented. First, the activities used did not suffer any grouping. Thus, the activities in their simplest state are, which makes it relevant to evaluate that the experiment carried out, if used in a large project, which may contain hundreds to thousands of activities, may require an evaluation in parts, for make your graphic demonstration viable.

The second issue to be evaluated is that the characteristics (variables) that were taken as a premise, are not limited to those that were used. Even more, since they are activities in their most unique and original state, other characteristics could be interesting for the manager, such as: equipment, regulatory standards (NRs) to be obeyed, number of employees, supplies and others. However, this level of detail demands from the manager and his team, both an alignment and an accurate process of recording the occurrences of each activity. Such a record can be strengthened in terms of effort, since activity by activity would be evaluated.

\subsubsection{Analysis With Groups By Levels}

In the second experiment, an analysis with grouped activities was proposed. This proposal is based on a very common activity in the projects carried out, which is the grouping of project management tools. Thus, a grouping by activity level was performed. The proposal is to group the activities up to the level 5, and the activities can be mentioned as Level 1 (Activity 1), Level 2 (Activity 1.1), Level 3 (1.1.1), Level 4 (1.1.1.1), Level 5 (1.1.1.1.1), that is, groups of activities were made up to the fifth level of task subdivision.

Leveled activities are available in Table 2. In this case, the methods Single (Appendix I.d), Average / Centroid (Appendix I.e) and Complete (Appendix I.f) were used.

Table 2: Examples Activities with Grouping (Level 5)

\begin{tabular}{|l|l|l|l|l|l|l|}
\hline Level & Activities & Code & Predominant area & duration & Disciplinarity & Num. of Disciplinarity \\
\hline 5 & Mechanical & 1 & 1 & 17 & 2 & 2 \\
\hline 5 & Electrical Detailed Engineering & 2 & 2 & 15 & 2 & 2 \\
\hline 5 & TA Detailed Engineering & 3 & 1 & 17 & 1 & 1 \\
\hline 5 & Fluid Interconnection & 36 & 4 & 40 & 1 & 1 \\
\hline 5 & Fluid services & 37 & 4 & 8 & 1 & 1 \\
\hline 5 & Electrical Interconnection & 38 & 2 & 42 & 1 & 1 \\
\hline
\end{tabular}




\section{Source: (Authors)}

In the grouped activities, it can be seen that activities 46 and 18 presented a relevant grouping compared to the others, in terms of time and discipline. A different meaning about this analysis is that it is not just individual activities analyzed, but a group of tasks that has been composed. Thus, this group of tasks will demand the distribution of resources, personnel and investments that may be presented.

\subsubsection{Analysis of the Focus Project}

With the results presented, a discussion becomes pertinent: the evaluated project presents serious deviations in time and financial resources. In addition, an aggravating factor, it presents, even if not standardized, the application of a project management methodology already recommended in the literature.

Thus, an assessment of the graphical analysis presented becomes pertinent: the graphs demonstrate a question of dissimilarity focused on time and disciplines, which makes it possible to focus on two assessments (including those of inspection).

The first is the dissimilarity in terms of runtime resources, which has a direct impact on financial issues. Activities with similar execution times should be more aligned and with shorter execution times, due to issues such as expertise, baggage and knowledge of the team and other factors that accelerate their execution.

Through the analysis carried out in section 3.3.1, analyzes on activities 1,2,3,17 and 18 can be carried out. With the proposed dissimilarity, it would be up to the manager to hold a meeting with the departments (represented by the disciplines employed in the activity) for a assessment of the reasons for such specific deadlines and times with a particular assessment of each activity. Regarding the analysis carried out in section 3.3.2, it would be possible to analyze macro characteristics, with a grouping, which could lose in terms of specificity, but gain in terms of the ability to be able to analyze projects with hundreds of activities like the one that is under analysis. In this case, activities 18 and 46 would be evaluated.

In both evaluations, it is pertinent to note that the analysis methodology could bring thousands of Brazilian Reais and days of savings in the execution of the project that already presented deviations. The second pertinent analysis is about the dissimilarity about the responsible sectors.

As the dissimilarity is also related to the characteristics of departments associated with the activities, an assessment (inspection) by departments, according to the set grouped via dendogram for activities of greater risk or dissimilarity, in order to control performance and resource expenditures. Thus, both by activities and by groups of activities, it was possible to make an analysis possible in terms of spending time and responsible sectors in a project that has characteristics of deviations from goals.

\section{Conclusion}

With the strategic readjustment it can be concluded that there are greater chances of success and accuracy for the planning and development of the project if applied the methodology of clusters with dendrogram. 
Previously, the order in which the activities were carried out was manually assigned by the Project Manager, who predicted the predecessor and successor tasks, and now, this destination will be achieved by grouping the activities of the teams using dendrograms that will define hierarchically the tasks.

It should be noted that the characteristics can be more detailed. In the experiments, four were used, however, through adequate control and data collection (in each of the process activities), more characteristics (variables) could be used, which would make the experiment via a dendogram with a higher accuracy.

The differential contribution of this paper, in relation to the works in the literature, is the demonstration of analysis of activities with and without grouping, in addition to an evaluation of a project with deviations, with a proposal for action to the manager on a project that may cause losses to an organization. Therefore, even using a project management methodology already disseminated in the literature, since the use of the FEL methodology was not complete, in addition to being totally dependent on the experience of a project team, which does not guarantee excellence in results in a project estimated in thousands of Brazilian Reais.

\section{References}

[1] Shmueli, G., Bruce, P. C., Yahav, I., Patel, N. R., and Lichtendahl Jr, K. C. (2017). Data mining for business analytics: concepts, techniques, and applications in R. John Wiley \& Sons.

[2] Murtagh, F., and Contreras, P. (2017). Algorithms for hierarchical clustering: an overview, II. Wiley Interdisciplinary Reviews: Data Mining and Knowledge Discovery, 7(6), e1219.

[3] Neto, J. C. A. d. S., Coutinho, Í., Teixeira, G., and de Moura, A. A. (Eds.). (2018). Measuring Maturity in Complex Engineering Projects. IGI Global.

[4] Pinto, J. S., Novaski, O., Anholon, R. And Besteiro, É. N. C. (2014) Proposta de método de mensuração da complexidade em projetos. Anais do III SINGEP - Simpósio Internacional de Gestão de Projetos, São Paulo, 2014.

[5] Dantas, D. C. (2018) "Uma Análise Multivariada do Valor Médio de Projetos Aprovados na Lei Rouanet, 2013-2017” Anais do Seminário Internacional de Estatística com R, v. 3, n. 1.

[6] Moraes, R. O. and Laurindo, F. J. B (2018) Multidimensionalidade da maturidade em gestão de projetos de sistemas de informação. Produto \& Produção, v. 9, n. 2. 
11th International Conference on Research in

ENGINEERING, SCIENCE \& TECHNOLOGY

Appendix I - Activity versus Dissimilarity

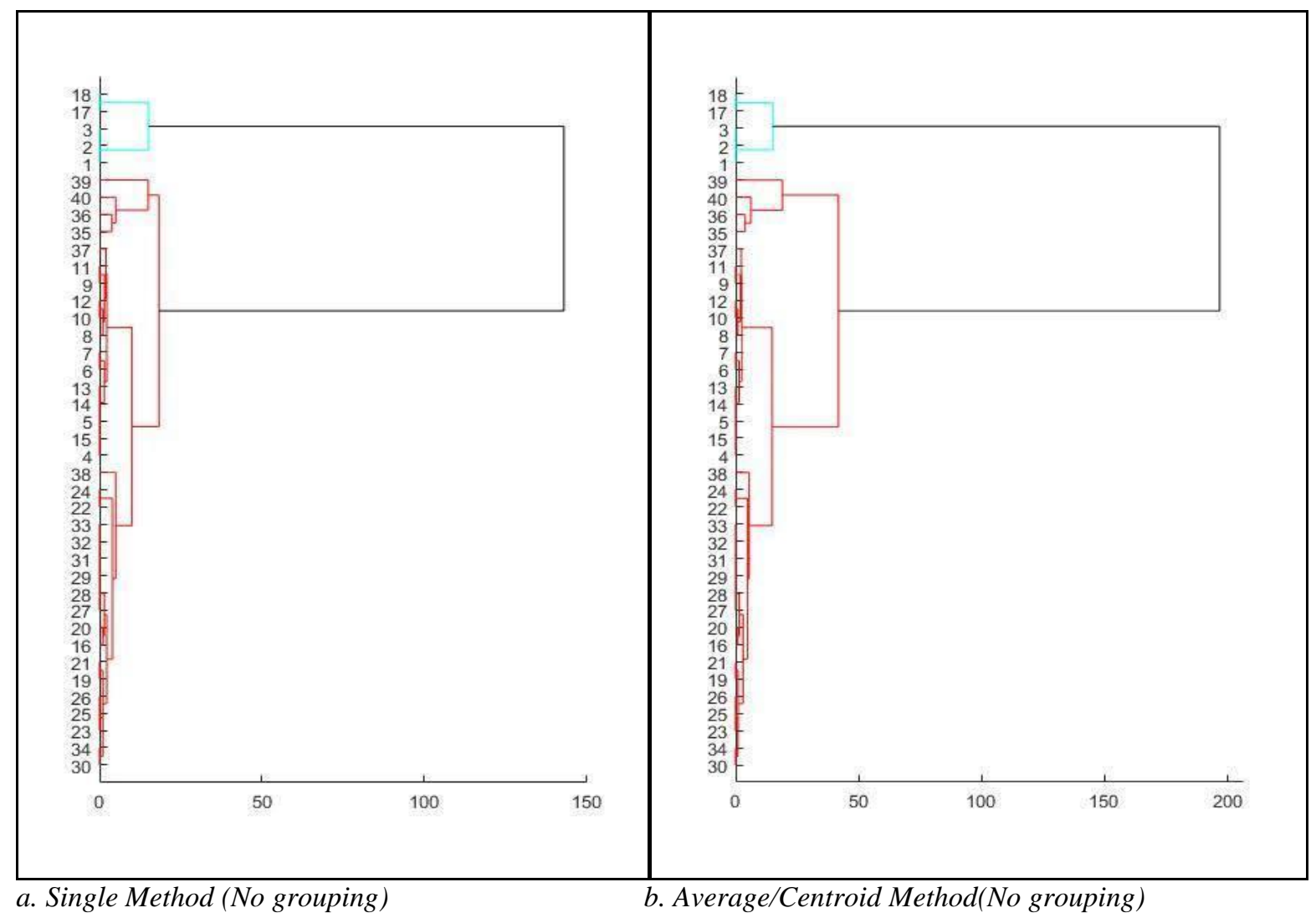


11th International Conference on Research in

ENGINEERING, SCIENCE \& TECHNOLOGY

Budapest, Hungary 19-21 March, 2021

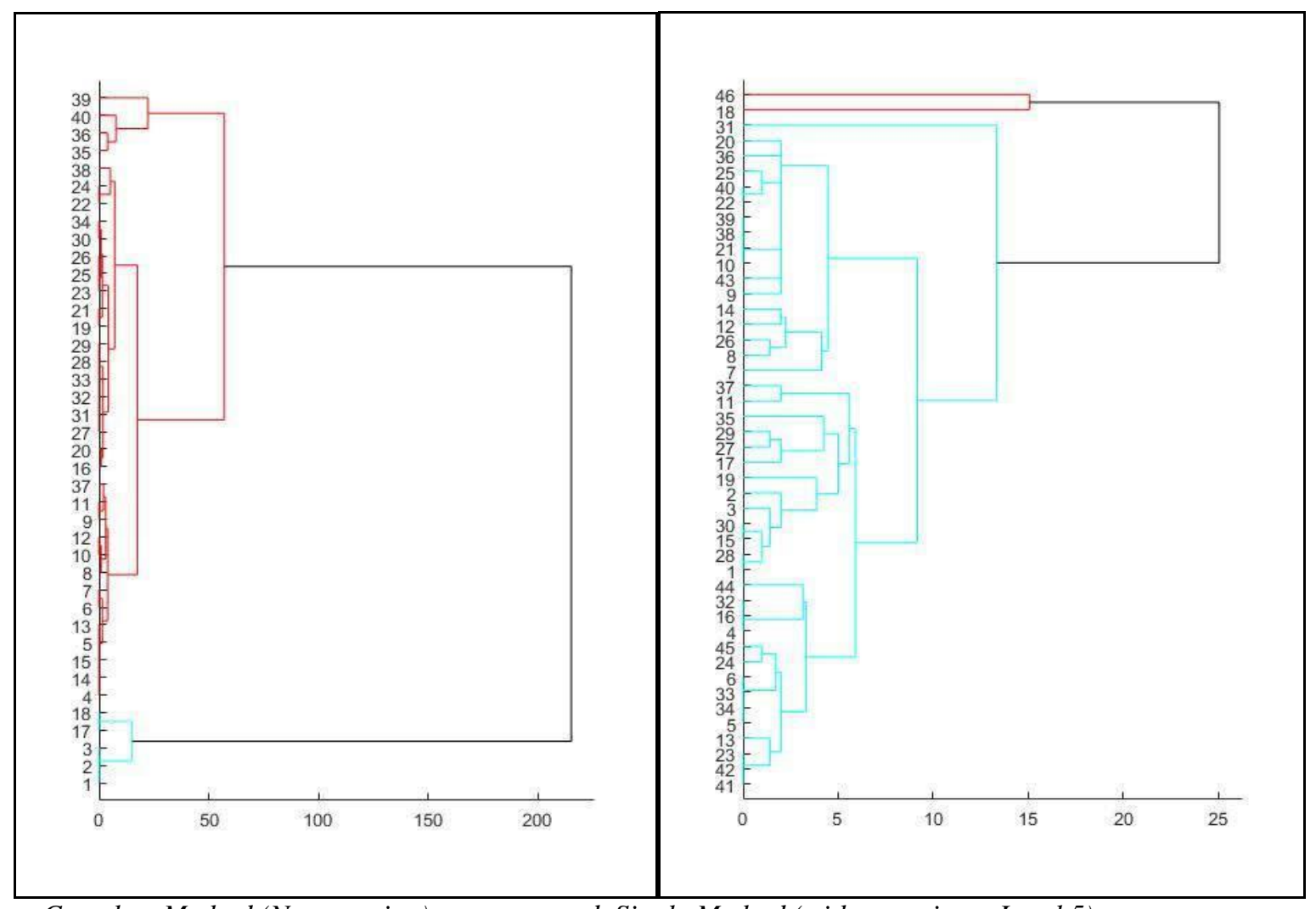

c. Complete Method (No grouping)

d. Single Method (with grouping - Level 5)

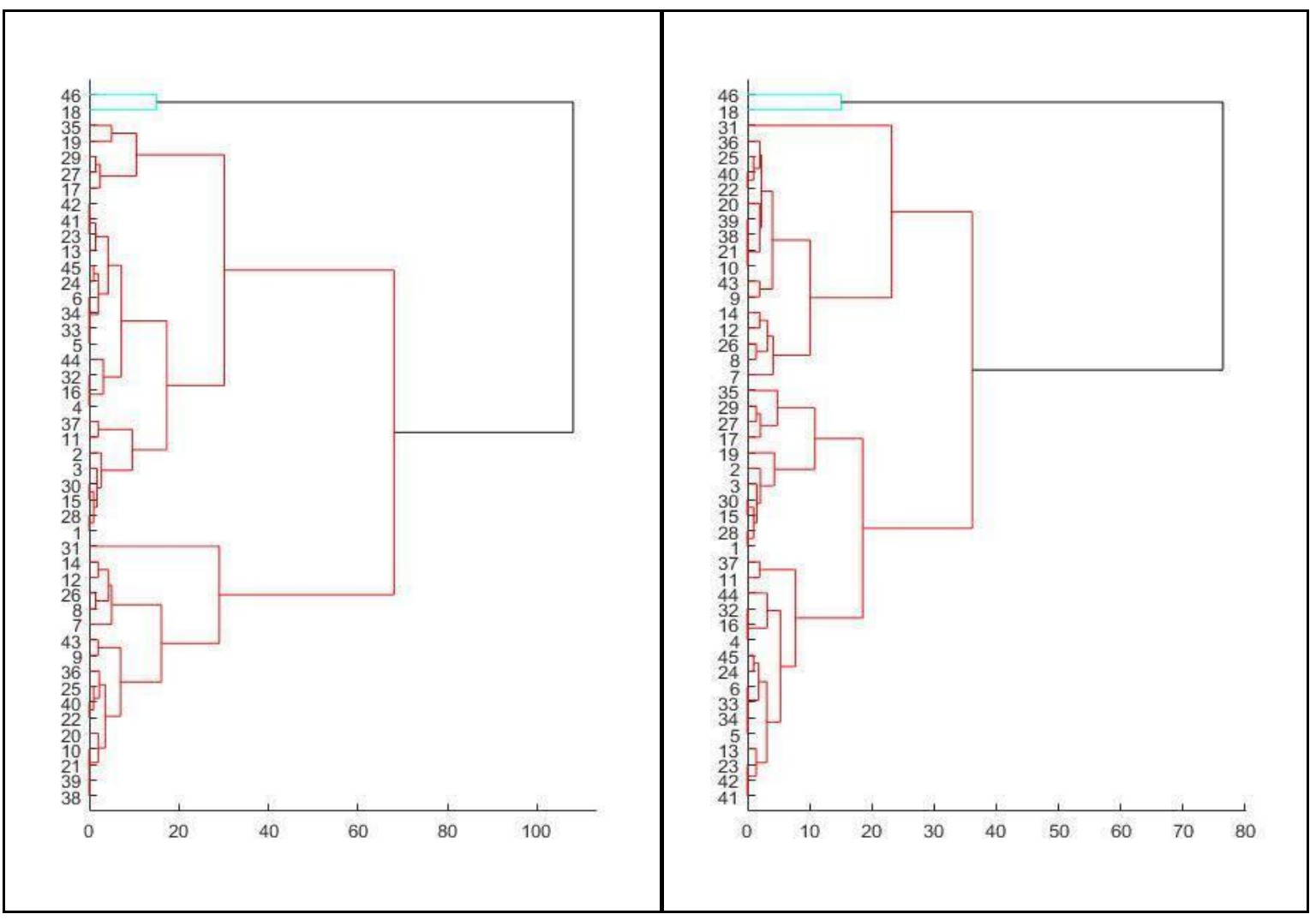


11 th International Conference on Research in

ENGINEERING, SCIENCE \& TECHNOLOGY

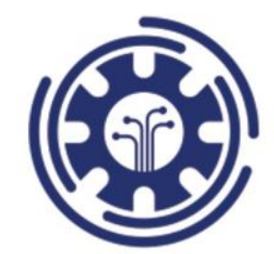

Budapest, Hungary

19-21 March, 2021

e. Average/Centroid Method ( with grouping - Level 5)f. Complete Method (with grouping - Level 5)

Source: (Authors) 